\title{
A clinical study of primary caesarean section at a tertiary care hospital in coastal city: an observational study
}

\author{
Chaitanya K. T., Lakshmi Manjeera M.*
}

Department of Obstetrics and Gynecology, K. S. Hegde Medical Academy, NITTE (Deemed to be University), Mangaluru, Karnataka, India

Received: 01 July 2020

Revised: 07 September 2020

Accepted: 08 September 2020

\section{*Correspondence:}

Dr. Lakshmi Manjeera M.,

E-mail: drmanjeera@nitte.edu.in

Copyright: (C) the author(s), publisher and licensee Medip Academy. This is an open-access article distributed under the terms of the Creative Commons Attribution Non-Commercial License, which permits unrestricted non-commercial use, distribution, and reproduction in any medium, provided the original work is properly cited.

\begin{abstract}
Background: Caesarean section represents the most significant operative intervention in obstetrics and its development and application has saved the lives of countless mothers and infants. However there has been a steady increase in the rate of caesarean sections worldwide. In this study, we aim to find the primary caesarean section rates and strategies to cut it down.

Methods: This was a prospective observational study done from January 2018 to June 2019 at Justice K. S. Hegde Charitable Hospital, Deralakatte, Mangaluru which included 379 cases of primary cesarean sections. All patients undergoing primary caesarean section with gestational age $>28$ weeks confirmed by dates, clinical examination and ultrasound were included in this study. Patients who had undergone previous caesarean section or were less than 18 years of age or were less than 28 weeks of gestation were excluded from the study.

Results: In this study the rate of primary caesarean section was found to be $14.5 \%$ and the majority of the study subjects belonged to the age group of 18-27 years $(62.3 \%)$. With respect to parity, primigravida were high in number (71.2\%), followed by multigravida. The number of emergency caesarean sections were (79\%) more than elective $(21 \%)$. The most common intra-operative complication was post-partum haemorrhage (PPH) in $2.8 \%$ and the most common postoperative complication was wound gape in $2.1 \%$.

Conclusions: Maximum efforts should be made to allow progression of vaginal delivery in primigravida which helps us to bring down the primary caesarean rate.
\end{abstract}

Keywords: Caesarean, Post-partum haemorrhage, Primigravida

\section{INTRODUCTION}

Caesarean delivery is one of the most commonly done major surgical procedures worldwide. ${ }^{1}$ The 2012 US Caesarean delivery rate was $32.8 \%$. The caesarean rate rose nearly to $60 \%$ from $1996(20.7 \%)$ to 2009 and has been stable since then. ${ }^{2}$ In India as per district level household survey 3 (DLHS) CS rate is $28.1 \%$ in private sector and $12 \%$ in public sector health facilities. ${ }^{3}$ Caesarean rates although sometimes are unnecessary, continue to increase like in the US where the rates were exceeding $27 \%$ in 2004 and did not show any signs of decrease. Indeed, there is growing discussion and acceptance of patient-choice caesarean section as legitimate birth option. ${ }^{4}$

The increase in caesarean section rate is becoming a major public health issue not only because it is a financial burden on health system and strain on families but also because it has long lasting effects on the health of both mother and baby and increases the overall caesarean section rates. It has been observed that caesarean deliveries are associated with increased risk of maternal and perinatal morbidity and postoperative complications as compared to vaginal deliveries. ${ }^{5}$ 
In the past 10 years, the rate of caesarean deliveries is increasing among the twin gestations. Perinatal outcomes for twin gestations in which the first twin is cephalic are not improved by caesarean delivery. Hence women with twin pregnancy with first cephalic presentation should be counselled to attempt vaginal delivery. ${ }^{6}$ A phenomenal rise in the Assisted Reproductive Techniques (ART) due to rising rate of infertility and advanced maternal age has also led to increase in caesarean sections. ${ }^{5}$

According to a study by Indian council of medical research (ICMR), the incidence of caesarean sections is 25.4\% for the years 1998-1999. Evidence from research studies shows there is a growing tendency for caesarean deliveries especially during complications confronted at the time of pregnancy and delivery. There is a trend of increase in caesarean sections as the maternal age increases along with parity. It is also observed that increase in maternal age will significantly elevate the risks of pregnancy. The indications for performing caesarean section have changed a lot in recent years and keep changing, for various circumstances. ${ }^{7}$

Primary caesarean section rate in teaching hospitals is rising due to technological advancement like ultrasound reporting of oligohydramnios, cord around neck, Doppler studies of absent or reversal of diastolic flow, infertility treatments, abnormal CTG and on maternal request. ${ }^{7}$

The rate of primary caesarean section is very important to be reduced because they lead to an increase in repeat caesarean section. It is particularly important to reduce the number of elective caesarean sections.

The present study is an effort to find out the leading indication for primary caesarean section and the various maternal and fetal morbidities in a tertiary hospital and try to find strategies to reduce the primary caesarean section rates.

\section{METHODS}

This is a Prospective Observational study conducted in a tertiary care hospital Justice K. S. Hegde Charitable Hospital, Deralakatte, Mangaluru. The study included a total of 379 cases that underwent primary caesarean section for various indications. The study period extended from January 2018 to June 2019.

\section{Inclusion criteria}

All patients undergoing primary caesarean section with gestational age $>28$ weeks confirmed by dates, clinical examination and ultrasound were included in the study.

\section{Exclusion criteria}

Those patients that have undergone previous caesarean sections were excluded from the study.
Written informed consent was sought from each of the study participants prior to inclusion in the study. The data was then collected using a piloted proforma meeting the objectives of the study by means of personal interview with the patient after taking informed consent.

The following parameters were studied:

Age, parity, BMI, indications, intra and post-operative complications, maternal and neonatal outcomes and the data was collected using the following inclusion and exclusion criteria.

Intra-operative complications include extension of uterine incision, uterine lacerations, atonic post-partum hemorrhage (PPH), traumatic $\mathrm{PPH}$, injuries to the adjacent structures, placenta accreta, etc.

Post-operative complications include PPH, shock, infections, intestinal obstruction, deep vein thrombosis, wound complications, secondary PPH.

Neonatal morbidity and mortality indicators include asphyxia, still birth, iatrogenic prematurity-leading to respiratory distress syndrome.

\section{Statistical analysis}

SPSS (Statistical Package for Social Sciences) version 20.0 [IBM SPASS statistics (IBM corp. Armonk, NY, USA released 2011)] was used to perform the statistical analysis. Data was entered in the excel spread sheet. Descriptive statistics of the explanatory and outcome variables was calculated by mean, standard deviation for quantitative variables, frequency and proportions for qualitative variables.

\section{RESULTS}

In our study there were a total of 2605 deliveries, out of which the total caesarean rate was $35.6 \%$ and the primary caesarean rate was $14.5 \%$.

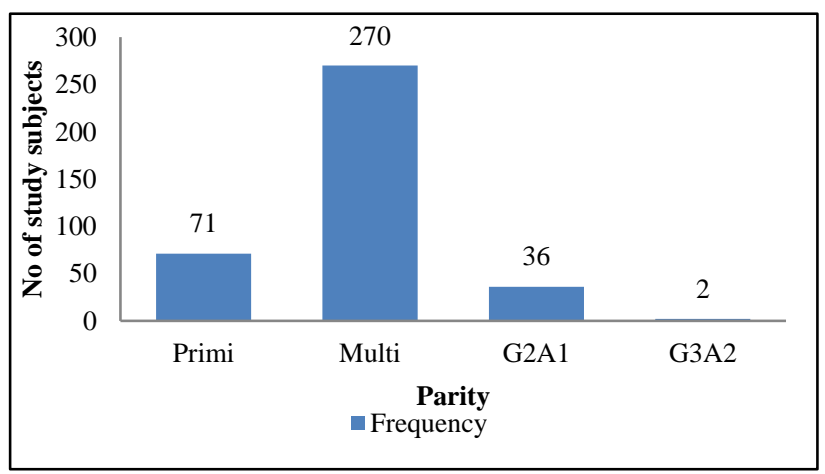

Figure 1: Parity of study participants.

In the present study, majority of the study subjects belonged to the age group of 18-27 years (62.3\%), 
followed by $28-35$ years $(33.5 \%)$ and $>35$ years $(4.2 \%)$. The mean age of the study subjects was $26.83 \pm 4.47$ years.

With respect to parity, primigravida were high in number (71.2\%), followed by multigravida (18.7\%), G2A1 in $9.5 \%$ and $\mathrm{G} 3 \mathrm{~A} 2$ in $0.5 \%$ (Figure 1 ).

Table 1: Age distribution of study participants.

\begin{tabular}{|lll|}
\hline Gestational age (in weeks) & Frequency & Percent \\
\hline $\mathbf{2 8 - 3 4}$ & 15 & 4.0 \\
\hline $\mathbf{3 4 - 3 7}$ & 44 & 11.6 \\
\hline $\mathbf{3 7 - 4 1}$ & 320 & 84.4 \\
\hline Total & 379 & 100.0 \\
\hline Mean GA & $38.55 \pm 1.91$ \\
\hline
\end{tabular}

Table 1 shows that the mean gestational age of the study subjects was $38.55 \pm 1.91$ weeks. Majority of the study subjects had gestational age of $37-41$ weeks $(84.4 \%)$, followed by 34-37 weeks (11.6\%) and 28-34 weeks (4\%).

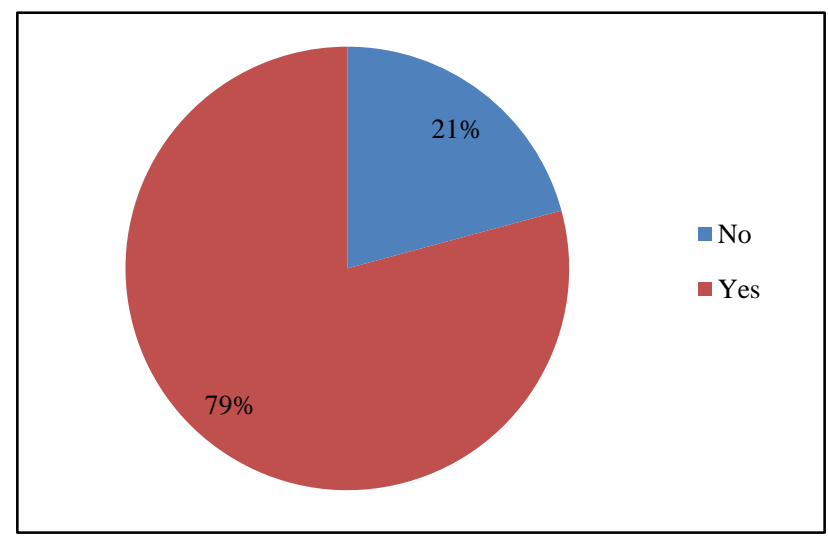

Figure 2: Patients undergoing emergency caesarean section.
Around $79 \%$ had emergency caesarean among the study subjects and around 21\% did not have emergency (Figure 2).

The most common indication was fetal distress in 66 cases, followed by malpresentation in 59 cases, meconium stained liquor in 57 cases, failed induction in 36 cases, CPD in 35 cases, non progression of labour in 29 cases, DTA in 26 cases (Table 2).

Around $94 \%$ of the babies did not have any complications. The most common neonatal complication was preterm/LBW in $2.6 \%$, followed by meconium aspiration syndrome in $1.3 \%$, HIE in $0.8 \%$ (Figure 4 ).

Table 2: Indications of caesarean sections.

\begin{tabular}{|lll|}
\hline Indications & Frequency & Percent \\
\hline Antepartum haemorrhage & 11 & 2.9 \\
\hline Others & 16 & 4.2 \\
\hline Failed induction & 36 & 9.5 \\
\hline Maternal request & 18 & 4.7 \\
\hline DTA (deep transverse arrest) & 26 & 6.9 \\
\hline Severe oligohydramnios & 10 & 2.6 \\
\hline $\begin{array}{l}\text { Altered Doppler/absent } \\
\text { diastolic flow }\end{array}$ & 3 & 0.8 \\
\hline Mal presentation & 59 & 15.6 \\
\hline Cord prolapse & 2 & .5 \\
\hline $\begin{array}{l}\text { CPD (cephalo-pelvic } \\
\text { disproportion) }\end{array}$ & 35 & 9.2 \\
\hline Fetal distress & 66 & 17.4 \\
\hline $\begin{array}{l}\text { Fetal distress + meconium } \\
\text { stained liquor }\end{array}$ & 1 & 0.3 \\
\hline Malpresentation & 17 & 4.5 \\
\hline $\begin{array}{l}\text { Meconium stained liquor in } \\
\text { early labour }\end{array}$ & 57 & 15.0 \\
\hline Non progression of labour & 29 & 7.7 \\
\hline Pre-eclampsia & 10 & 2.6 \\
\hline Total & 379 & 100.0 \\
\hline
\end{tabular}

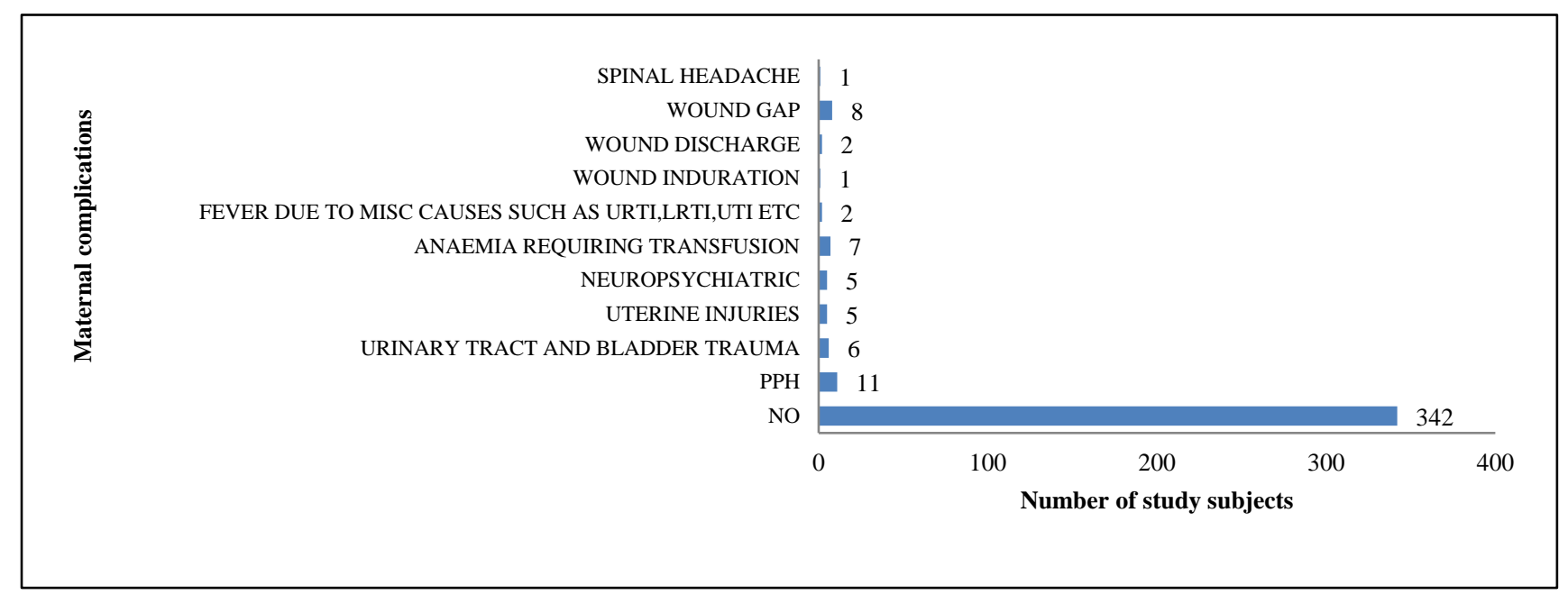

Figure 3: Maternal complications. 


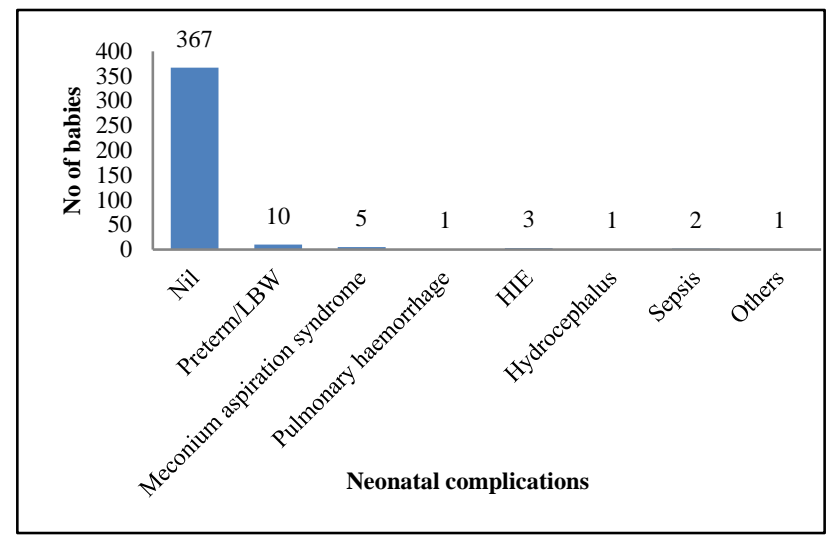

Figure 4: Neonatal complications.

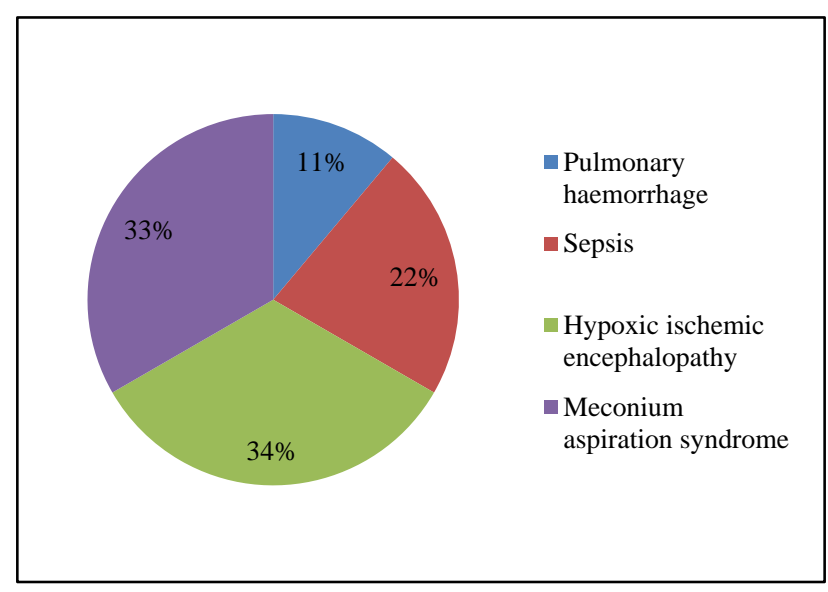

Figure 5: Causes for neonatal mortality.

\section{DISCUSSION}

In a study done by Datta et al, it showed that the maximum number of cases $(79.34 \%)$ had an age between 20-30 years which is very similar to the study done by Jain et al, Mande et al, by Rajbhandary et al, by Rao et al, Boyle et al as well as our study where the maximum age distribution was seen among 18-27 years of age $(62.3 \%)^{5,8,9,11-13}$ This shows that most of the primigravida who underwent caesarean section were in the age group of 20-30 years in most studies. Our study had 16 elderly primigravida accounting to $4.2 \%$ of the study population.

Our study showed that there were $71.2 \%$ primigravidae and the rest multigravidae. A study done by Jain et al showed that the primigravidae accounted for $72.4 \%$ and $27.6 \%$ were multigravidas which is similar to our study. ${ }^{11}$ Our study showed that most of the primary Caesarean sections were performed at 37-41 weeks of gestational age $(84.4 \%)$, followed by at $34-37$ weeks of gestational age $(11.6 \%)$. Preterm caesarean were seen only accounting to $4 \%$. This result correlates with the study done by Datta et al where the maximum primary caesarean were performed at 37-40 weeks of gestational age $(74.14 \%) .^{5}$
In our study the number of emergency primary caesarean was $79.2 \%$ compared to $20.8 \%$ which was done as elective cases. This result correlates with the study done by Datta et al, Jain et al and Moni et al and other studies where the emergency caesarean sections were higher than elective caesarean. In our study most of the elective caesarean was done for breech presentation.

The most common indications in our study was found to be fetal distress (32.1\%), malpresentations (16.7\%), deep transverse arrest (15.9\%), CPD (9\%) and non-progression of labour $(7.4 \%)$. The study done by Datta et al also shows a highest indication rate for fetal distress $(19.77 \%)$ followed by non-progression of labour (17.87\%) followed by altered Doppler values with severe FGR $(10.26 \%) .^{5}$ The study done by Jain et al and Rao et al, however, shows that maximum caesarean sections were done for malpresentation (34-40\%). ${ }^{11,12}$ All the other studies correlated with our study which showed fetal distress to be the most common indication for primary caesarean. The reason for fetal distress being the most common indication in our study may be due to the close intra partum continuous fetal monitoring which helped to pick up abnormal fetal heart rates and non-reassuring CTG.

In our study, PPH was found to be the most common maternal complication, which was similar to the findings observed in a study done by Datta et al, where PPH was seen in $5.2 \%$ of the study population. ${ }^{5}$

The most common neonatal complication observed was preterm seen in $2.6 \%$ of total cases followed by meconium aspiration syndrome (1.3\%) and hypoxic ischemic encephalopathy $(0.8 \%)$. The neonatal mortality rate was observed to be $0.2 \%$, i.e., there were a total of 9 neonatal deaths out of the 390 new born.

\section{CONCLUSION}

The number of emergency caesarean were more than elective caesarean. Fetal distress was the major indication in primary caesarean section. $\mathrm{PPH}$ and wound gape were the most commonly seen Intra-operative and postoperative complication. It is clear from our study that primary caesarean sections are not uncommon and that most of it is done for fetal sake. Various reasons like increased scientific advances, personal choice and medico legal considerations have led to an increased rate in caesarean sections. From our study it can be concluded that maximum trial should be given for vaginal delivery in primigravida which helps us to bring down the primary caesarean rate, this in turn helps us to cut down the overall caesarean section rate and surgery related complications. Following evidence-based labour protocols, auditing all the caesarean, regular antenatal check-ups, appropriate use of cardiotocography and patient education will also contribute in reducing the caesarean sections. 
Funding: No funding sources

Conflict of interest: None declared

Ethical approval: The study was approved by the Institutional Ethics Committee

\section{REFERENCES}

1. Stirrat G. The place of caesarean section. Contemp Rev Obstet Gynecol. 1998;10:177-83.

2. Martin JA, Hamilton BE, Osterman MJK, Curtin SC, Mathews TJ. Births: Final Data for 2012. Nat Vital Stat Rep. 2013;62(9):1-67.

3. Singh P, Hashmi G, Swain PK. High prevalence of caesarean section births in private sector health facilities- analysis of district level household survey4 (DLHS-4) of India. BMC Public Health. 2018;18(613):2-10.

4. US Department of Health and Human Services, National Institute of Health. Caesarean child birth. Publication no. 82-2067;1981.

5. Datta K, Singh P, Chopra N. Retrospective analysis of indications of primary caesarean sections done at a tertiary care hospital. Int J Reprod Contracept Obstet Gynecol. 2019;8:3161-7.

6. American College of Obstetricians and Gynecologists. Safe prevention of the primary cesarean delivery. Obstetric Care Consensus No. 1. Obstet Gynecol. 2014;123(3):693-711.

7. Sethi P, Vijaylaxmi S, Shailaja G, Bodhare T, Devi S. A study of primary cesarean section in multigravidae. Perspect Med Res. 2014;2:3-7.
8. Mande S, Kadam S, Dank G, Shiradakar S. Study of primary caesarean section in light of indications. Int J Recent Trends Sci Tech. 2016;17(3):241-4.

9. Rajbhanadary S, Shrivastava VR. Study of indications and post-operative complications of primary caesarean section in tertiary care hospital in Nepal. Int J Reprod Contracept Obstet Gynecol. 2018;7(3):835-40.

10. Moni M, Thanganadar AT, Yesubaktan SJ. A study on obstetric profile of mothers undergoing primary caesarean section and their neonatal outcome in a tertiary care centre, South Kerala. Int J Biomed Res. 2015;6(12):835-8.

11. Jain M, Patel A. A cross sectional study of rate, indications and complications of primary caesarean section. Int J Reprod Contracept Obstet Gynecol. 2016;5:1814-9.

12. Rao JH, Rampure N. Study of primary caesarean section in multiparous women. J Evol Med Dent Sci. 2013;2(24):4414-8.

13. Boyle A, Reddy UM, Landy HJ, Huang CC, Driggers RW, Laughon SK. Primary caesarean delivery in the United States. Obstet Gynecol. 2013;122(1):33-40.

Cite this article as: Chaitanya KT, Manjeera LM. A clinical study of primary caesarean section at a tertiary care hospital in coastal city: an observational study. Int J Reprod Contracept Obstet Gynecol 2020;9:4040-4. 